\title{
BMJ Open Exploring experiences of infertility amongst women and men in low- income and middle-income countries: protocol for a qualitative systematic review
}

Pooja Sripad, ${ }^{1}$ Sapna Desai (10 , ${ }^{2}$ Ricardo Regules, ${ }^{3}$ Suchandrima Chakraborty, ${ }^{2}$ Helen Habib (1) , ${ }^{4}$ Anny Rodriguez Viloria, ${ }^{5}$ Augustine Ankomah, ${ }^{4}$ Kamil Fuseini, ${ }^{4}$ Leah Jarvis, ${ }^{6}$ Karen Kirk, ${ }^{6}$ Nahla Tawab, ${ }^{7}$ Michelle Hindin ${ }^{6}$

To cite: Sripad P, Desai S, Regules R, et al. Exploring experiences of infertility amongst women and men in low-income and middle-income countries: protocol for a qualitative systematic review. BMJ Open 2021;11:e050528. doi:10.1136/ bmjopen-2021-050528

- Prepublication history and additional supplemental material for this paper are available online. To view these files, please visit the journal online (http://dx.doi.org/10.1136/ bmjopen-2021-050528)

PS and SD contributed equally.

Received 23 February 2021 Accepted 13 0ctober 2021

A) Check for updates

(C) Author(s) (or their employer(s)) 2021. Re-use permitted under CC BY-NC. No commercial re-use. See rights and permissions. Published by BMJ.

For numbered affiliations see end of article.

Correspondence to

Dr Sapna Desai;

sdesai@popcouncil.org

\section{ABSTRACT}

Introduction Infertility is increasingly recognised as a global public health issue for women and men that merits further investigation to support policy and programming. While research in high-income settings has examined the consequences of infertility and access to services, there has been limited synthesis of how individuals experience infertility in low-income and middle-income countries (LMICs). This protocol describes a systematic review that will synthesise qualitative evidence on experiences of infertility among women and men in LMICs.

Methods and analysis The review will follow the Enhancing transparency in reporting the synthesis of qualitative research (ENTREQ) guidelines for reporting on qualitative evidence syntheses. The study team will search for published literature in PubMed, CINAHL and Scopus and PsycINFO databases and review available grey literature. Using Covidence software, two independent reviewers will conduct title and abstract screening based on inclusion and exclusion criteria, followed by full-text reviews and extraction by a larger team. Quality will be appraised using an adapted version of the Critical Appraisal Skills Programme guidelines. We will conduct thematic synthesis to characterise individual experiences and related factors at the individual, interpersonal, community and health system levels. We will develop a conceptual framework to describe evidence on experiences of infertility in LMICs and to help inform interventions across settings.

Ethics and dissemination This protocol has been internally approved as exempt by the Institutional Review Board of the Population Council, as it does not involve contact with human subjects or personally identifying data Results of the review will be published in a peer-reviewed journal and will be used to inform future infertility research and programming in LMICs.

PROSPERO registration number CRD42021227742.

\section{BACKGROUND}

Infertility is an understudied reproductive health issue with wide-ranging consequences for
Strengths and limitations of this study

- First synthesis of qualitative evidence on experiences of infertility among women and men in low and middle-income countries (LMICs).

- Follows enhancing transparency in reporting the synthesis of qualitative research guidelines for synthesis of qualitative evidence.

- Draws from four databases and grey literature.

- Multicountry, multidisciplinary team across several LMIC settings.

- Excludes quantitative studies that may examine quality of life or treatment-seeking patterns.

women and men. A 2012 analysis of population data across 190 countries estimated that approximately $1.9 \%$ of women aiming to conceive (in ages 20-44) experienced primary infertility, along with $10.5 \%$ of women who have ever had a child (secondary infertility) ${ }^{1-3}$ This global estimate suggested that 48.5 million couples worldwide were affected by infertility, with the highest prevalence in South Asia, sub-Saharan Africa, North Africa/Middle East and Central/Eastern Europe. Further, this figure might be an underestimate influenced by underreporting and measurement issues. Despite posing a considerable burden on couples, infertility occupies a disproportionately low priority within sexual and reproductive health policy. Greater investment in programmes and evidence is required to improve the lives of those affected by infertility, particularly in low-resourced areas. ${ }^{4-6}$

The consequences of infertility can be devastating for women, particularly in settings where motherhood and reproduction are highly valued for social, cultural and economic reasons. ${ }^{6}$ Women affected by infertility may face stigma, shame, loss of social status, lower 
quality of life, marital stress and separation and negative economic consequences, as well as an increased risk of intimate partner violence. ${ }^{5-11}$ In contexts where childlessness is highly stigmatised, the fear of infertility can increase health risks, such as through avoiding contraception or not seeking medical care. ${ }^{12}$ While experiences vary by context, infertility is inevitably a gendered issue. ${ }^{611}$ Although research suggests that men and women may contribute equally to infertility, simple tests for males, such as sperm count and quality, are often not done. ${ }^{13}$ As a result, women typically shoulder a disproportionate burden of both medical interventions and social consequences of infertility. Previous research, mainly in high-income settings, has found that levels of distress and coping mechanisms also vary by gender. However, there has generally been less research on men's roles in the causes, diagnosis and treatment of infertility or the differential experiences of men and women. ${ }^{14} 15$

Understanding how individuals and couples experience infertility, and how it varies by context, is critical to developing interventions and influencing policy priorities. Examining experiences of infertility also uncovers influences and effects at the relationship, household and community levels beyond direct consequences. Underlying factors that may influence individual experience and treatment-seeking journeys include the legislative, health policy and systems environment that determine access, treatment and management options; peer, social and cultural norms; family and household dynamics, including childbearing attitudes, functional networks and support; and individual characteristics (ie, gender, wealth, education, ethnicity, disability and autonomy). ${ }^{5141617}$ Exploring experiences across contexts and populations can also provide insight into systems-level determinants, such as health systems capacity and access to interventions that influence how infertility is managed. ${ }^{17} 18$

There is a growing body of research that explores how men and women understand, cope with and address primary or secondary infertility in different settings. Research includes individual and collective narratives and life stories that reflect biomedical and emotional experiences, attitudes and beliefs regarding intervention and treatment, as well as the influence of context-specific norms related to reproduction and parenthood. ${ }^{19}{ }^{20}$ Research on infertility has largely been conducted in high income settings, with limited synthesis of individual experiences across low-income and middle-income countries (LMICs). ${ }^{3}{ }^{516}$ Recent reviews highlight the need to understand women and men's experiences in low-resource settings in particular, to identify areas for potential intervention and inform policy priorities in reproductive health more broadly. ${ }^{31417}$

\section{RESEARCH METHODOLOGY}

\section{Research objective and questions}

This systematic review aims to explore experiences of infertility among women and men in LMICs. Our research questions are:
- How do women and men experience (define, cope with and manage) infertility in low-income and middle-income settings?

- What factors at the individual, interpersonal, social and health systems levels influence how women and men experience infertility?

Our findings will be used to develop a conceptual framework to describe how men and women in LMICs experience infertility. Synthesis of men and women's experiences will also inform intervention development and programme implementation in LMICs. Review findings will likely also identify implications not only those experiencing infertility, but also for proximate actors such as families and health professionals.

\section{Review methods}

We will synthesise qualitative research that investigates women and men's experiences of infertility in LMIC. We will focus on individual perspectives and voices, in order to examine the diverse ways in which infertility is experienced, as well as expand how it is defined and conceptualised. Although evidence syntheses of infertility suggest the importance of employing a comprehensive, mixedmethods approach to understanding consequences of infertility, ${ }^{11}$ this review focuses on qualitative research as a first step to understand experiences as articulated by women and men directly. Qualitative methods are well suited to eliciting in-depth perspectives of individuals' experiences; they allow for individuals to recount a phenomenon (eg, infertility) to derive its meaning and reflect on how it fits into the broader narrative of their life experience. ${ }^{21}$

Criteria for included studies:

- Primary qualitative research studies that examine experiences of women and men of reproductive age (15-49 years) in LMIC. These may include qualitative observations and individual in-depth interviews, focus group discussions and key informant interviews conducted in facility and community settings, as independent studies or part of mixed-methods research.

- Research conducted in LMICs, according to World Bank classification. ${ }^{22}$

- Published between January 2000 and December 2020.

- Published in English, Spanish or French.

Exclusion criteria

- Studies that do not focus on infertility in their primary research objectives.

- Studies that do not examine individual experiences as defined above, that is, quality of life, interpersonal dynamics, social and economic consequences or access to infertility-specific and psychosocial services.

- Solely quantitative studies.

- Conference abstracts and dissertations.

The search will be conducted from January to March 2021, using Covidence software for both the screening and review. We anticipate study completion by December 2021. We will follow Preferred Reporting Items for Systematic Review and Meta-Analysis (PRISMA) 
Protocols guidelines in the search and selection process, and will report synthesised findings in accordance with the Enhancing transparency in reporting the synthesis of qualitative research (ENTREQ) guidelines for qualitative reviews. ${ }^{23}$ The review will not report on any identifying information and will synthesise information already in the public domain. This protocol has been exempted from review by the Institutional Review Board of the Population Council.

\section{Information sources}

We will search for published literature in PubMed, CINAHL and Scopus and PsycINFO, as well as grey literature found on organisational websites such as the Guttmacher Institute, Population Council, Infertility Society, WHO, ICRW, UNFPA, PSI, etc. In addition, we will search reference lists of previous studies and reviews.

\section{Search strategy}

Our search strategy includes a range of terms to capture individual experiences, such as stigma, economic and social costs and violence, and will be implemented across all databases. The PubMed search strategy is provided as an example (online supplemental appendix 1).

\section{Screening}

The screening across databases will be done using Covidence, a data management software that supports systematic reviews. Three reviewers will conduct title and abstract screening in the first stage. At the next stage, two reviewers will screen full text to identify included studies. A third reviewer will provide inputs to resolve any disagreements and to finalise selection.

\section{Appraisal}

We will use an adapted version of the Critical Appraisal Skills Programme guidelines to appraise the quality of included qualitative studies. ${ }^{24}$ We will not exclude studies based on quality, but will highlight gaps in evidence due to lack of confidence in findings.

\section{Extraction}

We will extract study characteristics in Covidence and a Microsoft Excel sheet. We will include information according to guidelines for qualitative evidence synthesis. ${ }^{24}$ For all studies, we will extract data on author, year, phenomena of interest, location and setting of the study, sampling procedures, sample size, and participants' gender and age. In addition, we will draw information on major findings and conclusions (online supplemental appendix 2 for extraction tool). We will extract information on theoretical orientation (ie, phenomenology, grounded theory, life history approach), and methods for data collection and analysis. We will also, if applicable, describe any interventions or programmes of focus within the study's scope (ie, content, who delivered it, how often). Data extraction will be conducted by a team of authors and collaboratively reviewed.

\section{Analysis}

We will conduct a thematic synthesis of literature to characterise men and women's experiences and the factors that affect these experiences, including but not restricted to, individual, interpersonal, community and health systems. We will use Microsoft Excel to organise data and conduct thematic analysis. Our final analysis, drawing from the thematic synthesis approach from Thomas and Harden ${ }^{25}$ will follow three stages: (1) inductive coding of text, using verbatim text of included papers, (2) generation of descriptive themes based on these codes across papers and (3) development of analytical themes that extend beyond primary studies, with a view to develop a conceptual framing of experiences of infertility.

\section{Ethics and dissemination}

This protocol has been internally approved as exempt by the Institutional Review Board of the Population Council, as it does not involve contact with human subjects or personally identifying data.

Our findings will be used to develop a conceptual framework to describe and analyse how men and women in LMICs experience infertility/subfertility. We anticipate this framework will help develop further quantitative and qualitative research studies as well as highlight implications for programmes and interventions. ${ }^{17}$ We plan to publish the findings in a peer-reviewed journal and share them through research communities, particularly within countries where the authors live and work.

\section{Patient and public involvement}

This systematic review of publicly available research did not involve patients or the public in the protocol design. We will ensure findings are disseminated with a diverse range of stakeholders across country settings.

\section{DISCUSSION}

To the best of our knowledge, this is the first systematic review to synthesise qualitative study findings on experiences of infertility in LMICs. We will examine findings from countries with an array of health systems, demographic, sociocultural and political contexts and publications in English, Spanish and French. Factors such as limited access to health services, stigmatisation and economic consequences may affect individuals' experiences differently both within and across settings. ${ }^{3} 1017$ We will examine how health systems and services respond to infertility across settings, such as how infertility experiences may provide insight into people-centredness of health systems. ${ }^{26}{ }^{27}$ Gender is a pivotal determinant in the how individuals experience infertility, both as culturally determined and informed by economic and social structures. ${ }^{514}$ Accordingly, we will examine our findings from the view of gender analysis within health systems as well as in the context of how economic, social and cultural factors shape people's experiences. ${ }^{26}$ Lastly, the process of integrating and synthesising findings will facilitate 
the development of an evidence-based and conceptually informed framework to describe how men and women in LMICs experience infertility. We hope the review will inform effective interventions, as well as elevate the understanding of this increasingly critical issue in public health. ${ }^{4}$

\section{Author affiliations}

${ }^{1}$ Population Council, Washington, DC, USA

${ }^{2}$ Population Council, New Delhi, India

${ }^{3}$ Population Council, Mexico City, Mexico

${ }^{4}$ Population Council, Accra, Ghana

${ }^{5}$ UCLA, Los Angeles, California, USA

${ }^{6}$ Population Council, New York, New York, USA

${ }^{7}$ Population Council Egypt, Cairo, Egypt

Acknowledgements We would like to acknowledge all the members of the Population Council's Strategic Initiative whose quarterly consultation aided in general feedback around the nature and scope of the systematic review. We thank Dr Jessica Gibson, University of California Los Angeles for her encouragement and feedback earlier in the process of considering the review scope.

Contributors PS, SD and RR conceputalised and drafted the protocol. SC, HH and ARV supported the development of the protocol, wrote pieces of the background, carried out preliminary searches to define search terms, and reviewed the manuscript. AA, KF, LJ, KK and NT provided substantive feedback to the manuscript. $\mathrm{MH}$ served as senior author, supported development of the protocol and provided feedback throughout. All authors reviewed and approved the final submission.

Funding Funding support for this review comes from the Population Council's Strategic Research Initiative investment (grant number N/A).

Competing interests None declared.

Patient consent for publication Not applicable.

Provenance and peer review Not commissioned; externally peer reviewed.

Supplemental material This content has been supplied by the author(s). It has not been vetted by BMJ Publishing Group Limited (BMJ) and may not have been peer-reviewed. Any opinions or recommendations discussed are solely those of the author(s) and are not endorsed by BMJ. BMJ disclaims all liability and responsibility arising from any reliance placed on the content. Where the content includes any translated material, BMJ does not warrant the accuracy and reliability of the translations (including but not limited to local regulations, clinical guidelines, terminology, drug names and drug dosages), and is not responsible for any error and/or omissions arising from translation and adaptation or otherwise.

Open access This is an open access article distributed in accordance with the Creative Commons Attribution Non Commercial (CC BY-NC 4.0) license, which permits others to distribute, remix, adapt, build upon this work non-commercially, and license their derivative works on different terms, provided the original work is properly cited, appropriate credit is given, any changes made indicated, and the use is non-commercial. See: http://creativecommons.org/licenses/by-nc/4.0/.

\section{ORCID iDs}

Sapna Desai http://orcid.org/0000-0003-2596-9726

Helen Habib http://orcid.org/0000-0001-7767-9312

\section{REFERENCES}

1 Mascarenhas $\mathrm{MN}$, Cheung $\mathrm{H}$, Mathers $\mathrm{CD}$, et al. Measuring infertility in populations: constructing a standard definition for use with demographic and reproductive health surveys. Popul Health Metr 2012;10:17.
2 Greil A, McQuillan J, Slauson-Blevins K. The social construction of infertility. Sociol Compass 2011;5:736-46.

3 Tanywe A, Matchawe C, Fernandez R, et al. Experiences of women living with infertility in Africa: a qualitative systematic review protocol. JBI Database System Rev Implement Rep 2018;16:1772-8.

4 Gipson JD, Bornstein MJ, Hindin MJ. Infertility: a continually neglected component of sexual and reproductive health and rights. Bull World Health Organ 2020;98:505-6.

5 Ombelet W, Cooke I, Dyer S, et al. Infertility and the provision of infertility medical services in developing countries. Hum Reprod Update 2008;14:605-21.

6 Inhorn MC. Right to assisted reproductive technology: overcoming infertility in low-resource countries. Int J Gynaecol Obstet 2009:106:172-4.

7 Tiu MM, Hong JY, Cheng VS, et al. Lived experience of infertility among Hong Kong Chinese women. Int J Qual Stud Health Wellbeing 2018:13:1554023.

8 Stellar C, Garcia-Moreno C, Temmerman M, et al. A systematic review and narrative report of the relationship between infertility, subfertility, and intimate partner violence. Int J Gynaecol Obstet 2016;133:3-8.

9 Dyer S, Lombard C, Van der Spuy Z. Psychological distress among men suffering from couple infertility in South Africa: a quantitative assessment. Hum Reprod 2009;24:2821-6.

10 Dyer SJ, Patel M. The economic impact of infertility on women in developing countries - a systematic review. Facts Views Vis Obgyn 2012;4:102.

11 Greil AL, Slauson-Blevins K, McQuillan J. The experience of infertility: a review of recent literature. Sociol Health IIIn 2010;32:140-62.

12 Boivin J, Carrier J, Zulu JM, et al. A rapid scoping review of fear of infertility in Africa. Reprod Health 2020;17:1-13.

13 Datta J, Palmer MJ, Tanton C, et al. Prevalence of infertility and help seeking among 15000 women and men. Hum Reprod 2016;31:2108-18.

14 Inhorn MC, Patrizio P. Infertility around the globe: new thinking on gender, reproductive technologies and global movements in the 21st century. Hum Reprod Update 2015;21:411-26.

15 Agarwal A, Mulgund A, Hamada A, et al. A unique view on male infertility around the globe. Reprod Biol Endocrinol 2015;13:37.

16 Rouchou B. Consequences of infertility in developing countries. Perspect Public Health 2013;133:174-9.

17 Passet-Wittig J, Greil AL. Factors associated with medical helpseeking for infertility in developed countries: a narrative review of recent literature. Soc Sci Med 2021;277:113782.

18 Chiware TM, Vermeulen N, Blondeel K, et al. Ivf and other art in lowand middle-income countries: a systematic landscape analysis. Hum Reprod Update 2021;27:213-28.

19 Inhorn M, Van Balen F. Infertility around the globe: new thinking on childlessness, gender, and reproductive technologies. Univ of California Press, 2002.

20 Laverty SM. Hermeneutic phenomenology and phenomenology: a comparison of historical and methodological considerations. Int $J$ Qual Methods 2003;2:21-35.

21 Creswell J. Qualitative inquiry and research design. Thousand Oaks, CA: SAGE Publications, 2007.

22 World Bank. World bank country and lending groups, 2021. Available: https://datahelpdesk.worldbank.org/knowledgebase/articles/906519world-bank-country-and-lending-groups [Accessed 24 Aug 2021].

23 Tong A, Flemming $\mathrm{K}$, Mclnnes $\mathrm{E}$, et al. Enhancing transparency in reporting the synthesis of qualitative research: ENTREQ. BMC Med Res Methodol 2012;12:181.

24 Noyes J, Booth A, Cargo M. Qualitative evidence. In: Cochrane Handbook for systematic reviews of interventions, 2019: 525-45.

25 Thomas J, Harden A. Methods for the thematic synthesis of qualitative research in systematic reviews. BMC Med Res Methodol 2008;8:1-10.

26 Morgan R, George A, Ssali S, et al. How to do (or not to do)... gender analysis in health systems research. Health Policy Plan 2016:31:1069-78.

27 Sheikh K, Ranson MK, Gilson L. Explorations on people centredness in health systems. Health Policy Plan 2014;29 Suppl 2:ii1-5. 